\title{
On arithmetic progressions having only few different prime factors in comparison with their length
}

\author{
by
}

\author{
Pieter Moree (Princeton, N.J.)
}

1. Introduction. For positive integers $a, d, k$ with $(a, d)=1$ and $k>2$ we will study the number of distinct prime factors of the integers in the arithmetic progression $a, a+d, \ldots, a+(k-1) d$. To fix notation, let $D_{a, d, k}=$ $a(a+d) \ldots(a+(k-1) d)$ and $\omega(a, d, k)=\omega\left(D_{a, d, k}\right)$ where, as usual, $\omega(n)$ is the number of distinct prime factors of the integer $n$.

By Dickson's prime $k$-tuplets Conjecture (a special case of Schinzel's Conjecture $\mathrm{H}$ [Ri, p. 312]), we expect that there are infinitely many integers $d$ for which each of the numbers $1+d, 1+2 d, \ldots, 1+(k-1) d$ is prime, so that $\omega(a, d, k)=k-1$ infinitely often for each $k$. On the other hand, it is easy to show (see the proof of Lemma 1) that if $\omega(a, d, k) \leq k-2$, then

$$
a(a+d) \leq(k-1) !
$$

so that $\omega(a, d, k) \leq k-2$ occurs only finitely often for each $k$. Inequality (1) has the important implication that in order to prove that an upper bound $\leq k-2$ holds with only finitely many exceptions $(a, d, k)$, it suffices to prove that $k$ is bounded from above (independently of $a$ and $d$ ).

The value of $\omega(1,2, k)$ is exactly $\pi(2 k)-1$, where $\pi(2 k)$ is the number of primes up to $2 k$, which is $\sim 2 k / \log k$ by the Prime Number Theorem. We thus see that $\omega(a, d, k)$ can be as small as a multiple of $k / \log k$ for at least one pair $(a, d)$ for each $k$. It is the purpose of this paper to study, in more detail, when $\omega(a, d, k)$ is less than some given multiple $k / \log k$ and to produce methods to determine all possible such triples $(a, d, k)$.

The following inequalities are due to Shorey and Tijdeman (see [ST1] respectively [ST2]),

$$
\omega(a, d, k) \geq \pi(k)
$$

Supported by the Dutch Organization for Scientific Research (NWO). 
and

$$
\omega(a, d, k) \geq k\left(1-\frac{\log k}{\log (a+(k-1) d)}\right)-1 .
$$

Provided $\omega(a, d, k) \leq k-2$, the latter inequality may be written as

$$
a+(k-1) d \leq k^{k /(k-1-\omega(a, d, k))} .
$$

It is a powerful result for, with this, one can deduce:

TheOREM 0. Fix a constant $\delta>0$. One can determine all triples $(a, d, k)$ of integers $a \geq 1, d \geq 2 e^{3 \delta}, k \geq 3,(a, d)=1$ for which

$$
\omega(a, d, k) \leq \min \left(k-2, \delta \frac{k}{\log k}\right) .
$$

Pro o f. If $k<e^{20 \delta}$ then, by (1), there are only finitely many possibilities for $a$ and $d$. So now assume $k \geq e^{20 \delta}$. Then $\omega \leq k / 20$ and $k \geq 20$. On using that $1 /(1-x) \leq 1+(4 / 3) x$ for $0<x \leq 1 / 10$, it follows that $k /(k-1-\omega)<$ $1+3 \omega / k$. Using (4) this gives that $a+(k-1) d \leq k^{1+3 \delta / \log k}=k e^{3 \delta}$, and so $d<2 e^{3 \delta}$.

The range for $d$ in Theorem 0 can be enlarged to something better than $d>e^{\delta}$.

Theorem 1. Fix a constant $\delta>0$. One can determine all triples satisfying (5) with $a \geq 1, k \geq 3,(a, d)=1$ and

$$
\delta<\log d+\sum_{p \mid d} \frac{\log p}{p-1} .
$$

Our main result will help us determine the critical bound for $\delta$ in Theorem 1.

THEOREM 2. Fix an integer $d \geq 2$ and define

$$
\varrho(d):=\frac{d}{\varphi(d)} \sum_{\substack{1 \leq m \leq d \\(m, d)=1}} \frac{1}{m} .
$$

For any given $\varepsilon>0$,

(i) there are only finitely many pairs $(a, k)$ for which

$$
\omega(a, d, k) \leq \min \left(k-2,(\varrho(d)-\varepsilon) \frac{k}{\log k}\right) ;
$$

(ii) if $k$ is sufficiently large and $1 \leq a<k$ with $(a, d)=1$ then

$$
\omega(a, d, k) \leq(\varrho(d)+\varepsilon) \frac{k}{\log k} .
$$


Note that

$$
\varrho(d)=\prod_{p \mid d}\left(1-\frac{1}{p}\right)^{-1} \sum_{\substack{m \leq d \\(m, d)=1}} \frac{1}{m}>\sum_{m \leq d} \frac{1}{m}>\log d .
$$

In Lemma 3 we show that

$$
\varrho(d)=\log d+\sum_{p \mid d} \frac{\log p}{p-1}+\gamma+R_{d}
$$

where $\gamma$ is Euler's constant and the "error term", $\left|R_{d}\right| \rightarrow 0$ as $d \rightarrow \infty$. Hence $\varrho(d)$ is asymptotically $\gamma(\approx .577)$ larger than the bound for $\delta$ in Theorem 1 .

By Theorem 2(ii) we observe that if $\varrho(d)<\delta$ for some $d$ then there will be infinitely many solutions to (5). We must therefore split the $d$ 's into two classes to give a more precise version of Theorem 1.

Corollary 1. Fix a constant $\delta>0$.

(i) There are only finitely many triples $(a, d, k)$ of integers with $a \geq 1$, $\varrho(d)>\delta, k \geq 3,(a, d)=1$ satisfying $(5)$.

(ii) For any given integers $a \geq 1, d \geq 2,(a, d)=1$, with $\varrho(d)<\delta$, there are infinitely many integers $k$ satisfying $(5)$.

Proof. (i) If $k-2 \leq \delta k / \log k$, then $k$ is bounded, whence, by (1), $a$ and $k$ are bounded. Otherwise, by Theorem 1 , we need only consider the triples $(a, d, k)$ with $d \leq e^{\delta}$. Hence we may assume that $d$ is fixed. Now we apply part (i) of Theorem 2 with $\varepsilon=\varrho(d)-\delta$.

(ii) Apply part (ii) of Theorem 2 with $\varepsilon=\delta-\varrho(d)$.

The appearance of the constant $\varrho(d)$ in Theorem 2 is not surprising. To illustrate this we consider the case $a<d,(a, d)=1$. Put $x=a+(k-1) d$ and denote the number of primes $\leq x$ satisfying $p \equiv a(\bmod d)$ by $\pi(x ; d, a)$. There are $\pi(x ; d, a)$ primes $p \equiv a(\bmod d)$ contributing to $\omega(a, d, k)$, and in general $\pi(x / m ; d, a / m)$ primes $p \equiv a / m(\bmod d)$ contributing to $\omega(a, d, k)$, where $1 \leq m \leq d$ and $(m, d)=1$. The set $\{a / m(\bmod d) \mid 1 \leq m \leq d$, $(m, d)=1\}$ forms a reduced residue system modulo $d$, since $(a, d)=1$. Applying the Prime Number Theorem for arithmetic progressions, which states that provided $(\alpha, d)=1$,

$$
\pi(x ; d, \alpha) \sim \frac{x}{\varphi(d) \log x} \quad(x \rightarrow \infty),
$$

we find that

$$
\omega(a, d, k) \sim \frac{x}{\varphi(d) \log x} \sum_{\substack{m \leq d \\(m, d)=1}} \frac{1}{m} \sim \frac{k}{\log k} \varrho(d) \quad(\text { as } k \rightarrow \infty) .
$$


The reason that we are not able to assert that we can effectively determine all triples $(a, d, k)$ in part (i) of Corollary 1 is that there is no explicit version of the Prime Number Theorem for arithmetic progression available as given by Rosser and Schoenfeld [RS1] for the Prime Number Theorem itself. McCurley has given some explicit result in case $d=3$ [Mc1] and for several other moduli in [Mc2]. Recently his work was improved by Ramaré and Rumely [RRu] using computations of zeros of $L$-functions by Rumely [Ru]. These results are very useful for determining all triples satisfying explicit inequalities related to (5). We shall describe a practical method for achieving this. As an illustration we shall establish the following conjecture of Shorey and Tijdeman on the cases in which equality in (1) holds:

TheOREM 3. The only solution to $\omega(a, d, k)=\pi(k)$ with $(a, d)=1, k \geq 4$ and $d>1$ is $\omega(1 \cdot 3 \cdot 5 \cdot 7 \cdot 9)=3=\pi(5)$.

Theorem 3 corresponds with $\delta=1$. Since $\varrho(d)>\log d$, it is easy to deduce that $\varrho(d) \geq 2$ for all $d \geq 2$, with equality only for $d=2$. Therefore if $\delta<2$ then, by Corollary 1(i), we deduce that there are only finitely many solutions to (5). The following result gives a precise description of the boundary case.

TheOREM 4. There are only finitely many triples $(a, d, k)$ with $(a, d)=1$ and $d \geq 2$, for which $\omega(a, d, k)<\pi(2 k-1)-1$. On the other hand $\omega(1,2, k)=$ $\pi(2 k-1)-1$ whenever $k \geq 3$.

The finitely many triples of Theorem 4 can be effectively computed, this is the content of Theorem $3^{\prime}$ of [Mo2, Chapter 7]. In the proof of this theorem the small values of $d$ create difficulties, in particular $d=3$. These problems are solved by using sharp estimates for the number of primes in arithmetic progressions with difference 1 and 3 and the corresponding $\theta$-functions. Following its line of proof an algorithm can be constructed to decide the conjecture of the author that $(1,3,10)$ is in fact the only such triple. Since one is dealing with the boundary case, one would expect that the computational effort in this case exceeds the computational effort needed to establish Theorem 1. Some preliminary work confirms this.

In Section 2 we derive a refinement of (1) and use it to prove Theorem 2(i) provided that $a>k$. This section is based on an elementary method due to Erdös. The Prime Number Theorem for arithmetic progressions is used in Section 3 to complete the proof of Theorem 2(i) and in Section 4 to prove Theorem 2(ii). Some more results of Erdős and a result from Section 1 are used to prove Theorem 1 . To prove Theorem 3 we follow the argument of the proof of Theorem 1, but make all estimates explicit. The proof of Theorem 4 is similar to that of Theorem 1.

For the proofs of $(2),(3)$ and further results on $\omega(a, d, k)$ see $[\mathrm{ST} 1,2,3,4]$. 
2. The case $a>d k$ of the proof of Theorem 2(i). The proof is based on the following refinement of inequality (1). The symbol $p$ will be exclusively used to denote primes.

LEMMA 1. Let $\theta_{d}(k)$ be the largest divisor of $(k-1)$ ! which is composed only of prime factors of $d$. Then

$$
a(a+d) \ldots(a+(k-1-\omega(a, d, k)) d) \leq \frac{(k-1) !}{\theta_{d}(k)} .
$$

Pro of. For every prime $p$ dividing $D_{a, d, k}$ leave out a term $a+i d$ such that $p^{m} \mid(a+i d)$ and that $p^{m+1}$ divides none of the other terms of the progression. In this way at most $\omega(a, d, k)$ terms are left out. The remaining terms are divisible only by primes less than $k$ coprime with $d$. Notice that the contribution of such a prime $p$ to the remaining terms is at most $p^{e(p)}$, where $e(p)$ is the exponent of $p$ in $(k-1)$ !, that is,

$$
e(p)=\sum_{i=1}^{\infty}\left[\frac{k-1}{p^{i}}\right] \text {. }
$$

Thus the product of the remaining terms is

$$
\leq \prod_{p \leq k-1,(p, d)=1} p^{e(p)}=\frac{(k-1) !}{\theta_{d}(k)} .
$$

On the other hand, the product of the remaining terms is at least $a(a+d) \ldots(a+(k-1-\omega(a, d, k)) d)$.

Corollary.

$$
a(a+d) \ldots(a+(k-1-\omega(a, d, k)) d) \leq(k-1) !
$$

and, if $\omega(a, d, k) \leq k-2$,

$$
a(a+d) \leq(k-1) !
$$

Inequality (7) will be called the Erdös inequality and inequality (6) the sharp form of the Erdös inequality. The Erdős inequality has the convenient property that its right hand side does not depend on $d$.

The contribution of $\theta_{d}(k)$ is estimated in the following lemma. Put

$$
\alpha(d)=d \prod_{p \mid d} p^{1 /(p-1)} .
$$

Lemma 2. For any given $d$ and $\varepsilon>0$, there exists an effectively determinable constant $k_{d}(\varepsilon)$ such that for every $k \geq k_{d}(\varepsilon)$,

$$
k\left(\log \left(\frac{\alpha(d)}{d}\right)-\varepsilon\right) \leq \log \theta_{d}(k) \leq(k-1) \log \left(\frac{\alpha(d)}{d}\right) .
$$


Proof. For every $n \in N$ and any prime $p$ we have

$$
\frac{(k-1)\left(1-p^{-n}\right)}{p-1}-n \leq \sum_{i=1}^{\infty}\left[\frac{k-1}{p^{i}}\right] \leq \frac{k-1}{p-1} .
$$

Using these inequalities and the identities

$$
\log \theta_{d}(k)=\sum_{p \mid d} \log p \sum_{i=1}^{\infty}\left[\frac{k-1}{p^{i}}\right] \quad \text { and } \quad \log \left(\frac{\alpha(d)}{d}\right)=\sum_{p \mid d} \frac{\log p}{p-1},
$$

the result is easily deduced. The upperbound is immediate, the lowerbound follows on taking $n, n \geq 1$, so large that $2^{-n}<\varepsilon /(2 \omega(d))<2^{1-n}$ (without loss of generality we may assume that $\varepsilon<2)$. Then

$$
\log \theta_{d}(k) \geq(k-1)\left(\log \left(\frac{\alpha(d)}{d}\right)-\frac{\varepsilon}{2}\right)-\log \left(\frac{4 \omega(d)}{\varepsilon}\right) / \log 2
$$

and the result follows with

$$
k_{d}(\varepsilon)=\frac{2 \log \left(\frac{\alpha(d)}{d}\right)+\frac{2}{\log 2} \log \left(\frac{4 \omega(d)}{\varepsilon}\right)}{\varepsilon} .
$$

The relation between $\alpha(d)$ and $\varrho(d)$ becomes clear in the next lemma.

Lemma 3. For every $d \geq 2$ and $\varepsilon>0$,

$$
\varrho(d)=\log \alpha(d)+\gamma+R_{d}=\log d+\sum_{p \mid d} \frac{\log p}{p-1}+\gamma+R_{d},
$$

with

$$
\left|R_{d}\right| \leq\left|R_{2}\right| \leq .0366 \quad \text { and } \quad\left|R_{d}\right|=O_{\varepsilon}\left(d^{\frac{\log 2+\varepsilon}{\log \log d}-1}\right) .
$$

Proof. Let $\mu$ denote the Möbius function. We have

$$
\begin{aligned}
\sum_{\substack{m \leq d \\
(m, \bar{d})=1}} \frac{1}{m} & =\sum_{m=1}^{d}\left\{\sum_{\delta \mid(m, d)} \mu(\delta)\right\} \frac{1}{m} \\
& =\sum_{\delta \mid d} \mu(\delta) \sum_{\substack{m=1 \\
\delta \mid m}}^{d} \frac{1}{m}=\sum_{\delta \mid d} \frac{\mu(\delta)}{\delta} \sum_{m=1}^{d / \delta} \frac{1}{m} \\
& =\sum_{\delta \mid d} \frac{\mu(\delta)}{\delta}\left(\log d-\log \delta+\gamma+\delta \frac{E_{2}}{d}\right),
\end{aligned}
$$

with $\left|E_{2}\right| \leq 2$. Here we use the fact that

$$
\sum_{m \leq d} \frac{1}{m}=\log d+\gamma+\frac{E_{1}}{d}, \quad \text { with }\left|E_{1}\right| \leq 2,
$$


which can be derived using Euler summation. On noticing that $\sum_{\delta \mid d} \mu(\delta) / \delta$ $=\varphi(d) / d$, we can rewrite the right hand side of (9) in the form

$$
\frac{\varphi(d)}{d}(\log d+\gamma)-\sum_{\delta \mid d} \frac{\mu(\delta)}{\delta} \log \delta+E_{3} \frac{2^{\omega(d)}}{d},
$$

with $\left|E_{3}\right| \leq 2$. Let $e(p)$ denote the exponent of $p$ in $d$. Then we have

$$
\begin{aligned}
\sum_{\delta \mid d} \frac{\mu(\delta)}{\delta} \log \delta & =\sum_{p \mid d} \log p \sum_{p \delta \mid d} \frac{\mu(p \delta)}{p \delta}=-\sum_{p \mid d} \frac{\log p}{p} \sum_{\delta \mid d p^{-e(p)}} \frac{\mu(\delta)}{\delta} \\
& =-\sum_{p \mid d} \frac{\log p}{p} \cdot \frac{\varphi\left(d p^{-e(p)}\right)}{d p^{-e(p)}}=-\frac{\varphi(d)}{d} \sum_{p \mid d} \frac{\log p}{p-1} .
\end{aligned}
$$

Inserting this in (10) and multiplying by $d / \varphi(d)$, we obtain on using the fact that $\left|E_{3}\right| \leq 2$,

$$
\left|R_{d}\right| \leq \frac{2^{\omega(d)+1}}{\varphi(d)}
$$

On using the estimate $\omega(d) \leq(\log d)(\log \log d)^{-1}(1+\varepsilon / 2)$, which holds for any given $\varepsilon>0$ for all $d$ sufficiently large, and the estimate $\varphi(d) \geq$ $(\log 2) d / \log (2 d)[\mathrm{Ri}, \mathrm{p} .172]$, we see that $\left|R_{d}\right|$ is of the required order. Note that $\left|R_{d}\right|$ tends to zero as $d$ tends to infinity.

To complete the proof we will show that

$$
\max _{d \geq 2}\left|R_{d}\right|=\left|R_{2}\right|=2-\log 4-\gamma \approx .0365 .
$$

For $d$ in $[3,30029]$ we first compute $2^{\omega(d)+1} / \varphi(d)$. In case this number exceeds .036 we compute $\left|R_{d}\right|$, otherwise we proceed to the next integer $d$. We find that $\left|R_{d}\right|$ is bounded above by .036 in this interval. Now assume $d \geq 30030(=2 \cdot 3 \cdot 5 \cdot 7 \cdot 11 \cdot 13)$. In case $\omega(d) \geq 6$, we have $2^{\omega(d)+1} / \varphi(d) \leq 2^{7} / \varphi(30030)<.036$. In case $\omega(d) \leq 5$ we have, by the estimate $\varphi(d) \geq(\log 2) d / \log (2 d)$ and the fact that $d / \log (2 d)$ is increasing for $d \geq 3$,

$$
\frac{2^{\omega(d)+1}}{\varphi(d)} \leq \frac{2^{6}}{\log 2} \cdot \frac{\log (60060)}{30030}<.036 .
$$

Thus the result follows.

R e m a r k 1 . At the cost of more computation, the above argument can be considerably simplified. The initial interval, for which we now take [3, 92600], is handled as before. For $d>92600$ we have, by the inequality $2 \sqrt{p} /(p-1)$ $<1$ valid for all $p \geq 7$,

$$
\left|R_{d}\right| \leq 2 \prod_{p^{e} \| d} \frac{2}{p^{e}-p^{e-1}}=\frac{2}{\sqrt{d}} \prod_{p^{e} \| d} \frac{2 p}{p^{e / 2}(p-1)}
$$




$$
\leq \frac{2}{\sqrt{d}} \prod_{p^{e} \| d} \frac{2 \sqrt{p}}{p-1} \leq \frac{2 \sqrt{30}}{\sqrt{d}}<.036 .
$$

R e mark 2. Lemma 3 sharpens Lemma 3 of Granville [G], who gives an estimate for $\varrho(d)$ with error term $O(d \omega(d) / \varphi(d))$.

Theorem 5. Let $c>0$ and $\varepsilon>0$ be fixed. Let $d \geq 2$ be a fixed integer. One can determine all pairs $(a, k)$ with $a>c d k, \omega(a, d, k) \leq k-2$ and

$$
\omega(a, d, k) \leq\left[\log \alpha(d)+\log c+(c+1) \log \left(1+\frac{1}{c}\right)-\varepsilon\right] \frac{k}{\log k} .
$$

Proof. Let $f(k)$ denote the greatest integer part of the right hand side of (11). Take $k_{0}$ so large that $f(k) \leq k-2$ for $k \geq k_{0}$. Assume that the triple $(a, d, k)$ with $k \geq k_{0}$ satisfies (11). Using the sharp form of the Erdös inequality (6) it follows that

$$
\begin{aligned}
& \frac{(a+d(k-1-f(k))) !}{(a-d) !} \\
& \quad \leq\{a(a+d) \ldots(a+d(k-1-f(k)))\}^{d} \leq\left(\frac{(k-1) !}{\theta_{d}(k)}\right)^{d} .
\end{aligned}
$$

Notice that the left hand side of (12) is monotonically increasing in $a$. Using the estimate $\log k !=k \log k-k+O(\log k)$, Lemma 2 in the weaker form $\log \theta_{d}(k)=k \log (\alpha(d) / d)+o_{d}(k)$ and taking for $a$ the smallest integer $\geq c k d$ it follows that

$$
\begin{aligned}
\log \{[a+d(k & -1-f(k))] !\} \\
& =d k\left[(c+1) \log [k d(c+1)]-c-1-\frac{f(k) \log k}{k}+o_{d}(1)\right]
\end{aligned}
$$

and

$$
\begin{aligned}
\log [(a-d) !\{( & \left.k-1) !\}^{d} \theta_{d}(k)^{-d}\right] \\
& =d k\left[c \log (c d k)+\log k-c-1-\log \left(\frac{\alpha(d)}{d}\right)+o_{d}(1)\right] .
\end{aligned}
$$

From this and (12) it follows that

$$
f(k) \frac{\log k}{k}+o_{d}(1) \geq \log \alpha(d)+\log c+(c+1) \log \left(1+\frac{1}{c}\right) .
$$

So there are only finitely many possibilities for $k$. Since by assumption $\omega(a, d, k) \leq k-2$, it follows by (1) that there are only finitely many possibilities for $a$. In order to prove that the finitely many pairs $(a, k)$ can be determined, it suffices to show that the ineffective estimates occurring, $\log k !=k \log k-k+O(\log k)$ and $\log \theta_{d}(k)=k \log (\alpha(d) / d)+o_{d}(k)$, can be made effective. Since $\log (k !)$ is in $[k \log k-k,(k+1) \log k]$ for $k \geq 7$ and 
$\theta_{d}(k)$ can be effectively estimated from above and below by Lemma 2 , this is indeed the case.

Corollary. Let $d \geq 2$ be a fixed integer. One can determine all pairs $(a, k)$ with $a>d k$ and

$$
\omega(a, d, k) \leq \min \left(k-2,(\varrho(d)+.76) \frac{k}{\log k}\right) .
$$

Proof. We apply Theorem 5 with $c=1$. By Lemma 3 we have

$$
\log \alpha(d)>\varrho(d)-\gamma-.04>\varrho(d)-.62 .
$$

3. The case $a \leq d k$ of the proof of Theorem 2(i). We show in Lemma 4 that all primes in a certain union of finite arithmetic progressions divide $D_{a, d, k}$. Then we apply the Prime Number Theorem for arithmetic progressions. First we introduce some notation.

If $j$ is coprime to $d$ we denote by $\pi(x ; d, a / j)$ the number $\pi(x ; d, c)$ where $c$ is any integer such that $c j \equiv a(\bmod d)$. By $\sum_{j \leq n}^{(d)}$ we denote the sum over the positive integers $j \leq n$ with $(j, d)=1$. Again we write $x=a+(k-1) d$.

Lemma 4. Put $n=\min (d, a / k)$. If $k>d$ then

$\omega(a, d, k) \geq \sum_{j \leq d}^{(d)} \pi\left(\frac{x}{j} ; d, \frac{a}{j}\right)-\sum_{j \leq n}^{(d)} \pi\left(\frac{a}{j}-1 ; d, \frac{a}{j}\right)+\sum_{j \leq n}^{(d)} \pi\left(k-1 ; d, \frac{a}{j}\right)$.

Proof. If $p$ is a prime with $p<k$ and $p \nmid d$, then $p \mid D_{a, d, k}$. Therefore there are at least $\pi(k-1)-\omega(d)$ primes less than $k$ which divide $D_{a, d, k}$. Note that the elements of $\{a / j: 1 \leq j \leq d,(j, d)=1\}$ form a complete system of primitive residues mod $d$, since $(a, d)=1$. Hence

$$
\pi(k-1)-\omega(d)=\sum_{j \leq d}^{(d)} \pi\left(k-1 ; d, \frac{a}{j}\right) .
$$

Let $j$ be an integer with $1 \leq j \leq d$ and $(j, d)=1$. Let $p \geq k$ be a prime such that $p \equiv a / j(\bmod d)$ and $a / j \leq p \leq x / j$. Then $j p \equiv a(\bmod d)$ and $a \leq j p \leq x$, whence $p \mid D_{a, d, k}$. The number of primes $p \geq k$ with $p \equiv a / j$ $(\bmod d)$ and $p \mid D_{a, d, k}$ is therefore at least

$$
\pi\left(\frac{x}{j} ; d, \frac{a}{j}\right)-\pi\left(\frac{a}{j}-1 ; d, \frac{a}{j}\right) \quad \text { if } a \geq j k
$$

and

$$
\pi\left(\frac{x}{j} ; d, \frac{a}{j}\right)-\pi\left(k-1 ; d, \frac{a}{j}\right) \quad \text { if } a<j k .
$$


It follows that the number of primes $p \geq k$ dividing $D_{a, d, k}$ is at least

$$
\sum_{j \leq d}^{(d)} \pi\left(\frac{x}{j} ; d, \frac{a}{j}\right)-\sum_{j \leq n}^{(d)} \pi\left(\frac{a}{j}-1 ; d, \frac{a}{j}\right)-\sum_{a / k<j \leq d}^{(d)} \pi\left(k-1 ; d, \frac{a}{j}\right),
$$

On combining both lower bounds, the lemma follows.

Proof of Theorem 2(i). We distinguish three cases. Without loss of generality we may assume that $k$ is sufficiently large.

(a) $a<k$. Then by Lemma 4 ,

$$
\begin{aligned}
\omega(a, d, k) & \geq \sum_{j \leq d}^{(d)} \pi\left(\frac{x}{j} ; d, \frac{a}{j}\right) \sim \frac{x}{\varphi(d) \log x} \sum_{j \leq d}^{(d)} \frac{1}{j} \\
& \geq \frac{(k-1) d}{\varphi(d) \log (k(d+1))} \sum_{j \leq d}^{(d)} \frac{1}{j} \gtrsim \frac{k}{\log k} \varrho(d) .
\end{aligned}
$$

(b) $k<a \leq k d$. By Lemma 4 again,

$$
\begin{aligned}
\omega(a, d, k) & \geq \sum_{j \leq d}^{(d)} \pi\left(\frac{x}{j} ; d, \frac{a}{j}\right)-\sum_{j \leq d}^{(d)} \pi\left(\frac{a}{j}-1 ; d, \frac{a}{j}\right) \\
& \gtrsim \frac{1}{\varphi(d)}\left\{\sum_{j \leq d}^{(d)} \frac{x / j}{\log (x / j)}-\sum_{j \leq d}^{(d)} \frac{a / j}{\log (a / j)}\right\} \\
& \gtrsim \frac{1}{\varphi(d)} \sum_{j \leq d}^{(d)} \frac{(x-a) / j}{\log k}=\frac{k-1}{\log k} \varrho(d),
\end{aligned}
$$

where in the derivation of the latter asymptotic inequality we use the fact that $a / k, k / a, x / k$ and $k / x$ are all bounded from above.

(c) $a>k d$. Apply the Corollary to Theorem 5 .

\section{Proof of Theorem 2(ii)}

Lemma 5. If $a<k$ and $d<k$, then

$$
\omega(a, d, k)=\sum_{j \leq d}^{(d)} \pi\left(\frac{x}{j} ; d, \frac{a}{j}\right) .
$$

Pr o of. In view of Lemma 4 we need only prove the $\leq$ case. If the prime $p \geq k$ divides $D_{a, d, k}$, then there is precisely one $i$ with $0 \leq i<k$ and one integer $j$ such that $j p=a+i d$. By $(a, d)=1$, we have $(j, d)=1$. Since $a<k$, we have $j p<k(d+1)$ whence $j \leq d$. It follows that $p \equiv a / j(\bmod d)$ and $k \leq p \leq x / j$. Therefore the total number of primes $p \geq k$ dividing $D_{a, d, k}$ is at most

$$
\sum_{j \leq d}^{(d)}\left(\pi\left(\frac{x}{j} ; d, \frac{a}{j}\right)-\pi\left(k-1 ; d, \frac{a}{j}\right)\right) .
$$


Every prime $p<k$ dividing $D_{a, d, k}$ is coprime to $d$. Hence the number of such primes is at most

$$
\sum_{j \leq d}^{(d)} \pi\left(k-1 ; d, \frac{a}{j}\right)
$$

Proof of Theorem 2(ii). Let $d$ be any positive integer. Let $k$ be any "large" positive integer. Let $a<k$. Then

$$
\begin{aligned}
\omega(a, d, k) & =\sum_{j \leq d}^{(d)} \pi\left(\frac{x}{j} ; d, \frac{a}{j}\right) \sim \sum_{j \leq d}^{(d)} \frac{x / j}{\log (x / j)} \cdot \frac{1}{\varphi(d)} \\
& \lesssim \frac{x}{\log x} \cdot \frac{1}{\varphi(d)} \sum_{j \leq d}^{(d)} \frac{1}{j} \lesssim \frac{k}{\log k d} \varrho(d) \leq \frac{k}{\log k} \varrho(d) .
\end{aligned}
$$

5. Proof of Theorem 1. As in the proof of the Theorem 0 we can show that $d$ is bounded. Hence we may assume that $d$ is fixed. Recall that if we can find an upper bound for $k$ then, by (1), $a$ and $d$ are bounded. So we may assume that $k$ is large. Again we write $\alpha(d)=d \prod_{p \mid d} p^{1 /(p-1)}$. Further we put

$$
P_{k}(a, d)=\frac{1}{k !} \prod_{p \mid d} p^{[k /(p-1)]} \prod_{m=1}^{k}(a+m d) .
$$

We shall use the following elementary results due to Erdős.

Lemma 6. Let $a<d$. Then

(i) $P_{k}(a, d)$ is an integer.

(ii) If $p^{m} \| P_{k}(a, d)$ then $p^{m} \leq(k+1) d$.

(iii) $P_{k}(a, d)>(\alpha(d))^{k} / d$.

Proof. See Erdős [E].

We observe that for $k \geq d$ the prime decompositions of $P_{k}(a, d)$ and $\prod_{m=1}^{k}(a+m d)$ differ only for the prime factors $\leq k$. We shall use Lemma 6 to estimate the number $\omega_{1}$ of distinct prime factors $p \geq k$ in $D_{a, d, k}$. This will yield the following result.

Lemma 7. Let $\delta>0$ be fixed. One can determine all triples satisfying

$$
\omega(a, d, k) \leq \min \left(k-2, \delta \frac{k}{\log k}\right)
$$

with $a \geq 1, k \geq 3,(a, d)=1, a<d$ and $\delta<\log \alpha(d)$. 
Proof. Let $\varepsilon>0$. Using Lemma 6(ii) and $\sum_{p \leq x} \log p \sim x$ we find

$$
\begin{aligned}
P_{k}(a, d) & \leq \prod_{p \leq \sqrt{(k+1) d}}(k+1) d \prod_{\sqrt{(k+1) d}<p \leq k} p \prod_{\substack{p>k \\
p \mid D_{a, d, k}}} p \prod_{p \mid a+k d} p \\
& \leq((k+1) d)^{\sqrt{(k+1) d}} e^{(1+\varepsilon) k}(k d)^{\omega_{1}} \quad \text { for } k \text { large. }
\end{aligned}
$$

Hence by Lemma 6(iii),

$$
\omega_{1} \log (k d) \geq k(\log \alpha(d)-1-\varepsilon)-\log d-\sqrt{(k+1) d} \log ((k+1) d) .
$$

This implies, for all $k$ sufficiently large

$$
\omega_{1} \geq \frac{k}{\log k}(\log \alpha(d)-1-2 \varepsilon) .
$$

The number of primes $p<k$ dividing $D_{a, d, k}$ equals

$$
\omega(a, d, k)-\omega_{1}=\pi(k-1)-\omega(d) \geq \frac{k}{\log k}(1-\varepsilon),
$$

for all $k$ sufficiently large. Hence, for all $k$ sufficiently large,

$$
\omega(a, d, k) \geq \frac{k}{\log k}(\log \alpha(d)-3 \varepsilon) .
$$

Choose $\varepsilon<(1 / 3)(\log \alpha(d)-\delta)$ and take $k$ so large that the latter inequality for $\omega(a, d, k)$ holds. Then we have a contradiction with (5). Thus $k$ is bounded.

Next we relax the condition on $a$.

Lemma 8. The statement of Lemma 7 is still true if the condition $a<d$ is replaced by $a<k$.

Proof. Suppose $a, d$ and $k$ are such that (5) holds with $a \geq 1, k \geq 3$, $(a, d)=1, a<k$ and $\delta<\log \alpha(d)$. Let $b$ be such that $b \equiv a(\bmod d), 0<b \leq d$. Put $l=(a-b) / d$. Every prime factor of $b(b+d) \ldots(a-d)$ is less than $k$ and coprime to $d$ and therefore a prime factor of $a(a+d) \ldots(a+(k-1) d)=D_{a, d, k}$. It follows that

$$
\omega(a, d, k)=\omega(b, d, k+l) \geq \omega(b, d, k) .
$$

Notice that the triple $(b, d, k)$ was already found in Lemma 7 . Since $k$ is therefore bounded and $a<k$, we can determine all triples $(a, d, k)$ which satisfy the conditions of Lemma 8.

Proof of Theorem 1. Recall that we may assume that $d$ is fixed. By Lemma 8 we can determine all triples $(a, d, k)$ with $a<k$ which satisfy the conditions of Theorem 1 . If $a \geq k$ then we apply Theorem 5 with $c=1 / d$ and use the fact that $\varepsilon:=\log \frac{1}{d}+\left(\frac{1}{d}+1\right) \log (1+d)>0$ for every $d \geq 1$. 
6. Proof of Theorem 4. Suppose $(a, d, k)$ satisfies

$$
\omega(a, d, k)<\pi(2 k-1)-1 .
$$

It suffices to prove that $k$ is bounded. By (4) we have

$$
a+d(k-1) \leq k^{\frac{k}{k-1-\omega(a, d, k)}} \leq k^{1+\frac{\pi(2 k-1)}{k-\pi(2 k-1)}} .
$$

Using the Prime Number Theorem we deduce that the right hand side of the latter inequality divided by $k-1$ is $<8$ for every $k$ sufficiently large, and so in this case $d \leq 7$. For $d=3, \ldots, 7$ and $k$ sufficiently large, $\pi(2 k-1)-2$ $\leq 2.24 k / \log k<\frac{9}{4} k / \log k \leq \varrho(d) k / \log k$ and so by Theorem $2(\mathrm{i})$ the integers $k$ such that (14) is satisfied for some $a$ with $\omega(a, d, k)<\pi(2 k-1)-1$ are bounded above. So it remains to show that if $(a, 2, k)$ satisfies (14), then $k$ is bounded above. In the case $a \leq k+1$ we have $\omega(a, 2, k)=\pi(a+2(k-1))-1 \geq$ $\pi(2 k-1)-1$ and there are no solutions. So suppose that $a \geq k+2$. By Theorem 5 the $k$ satisfying $\omega(a, 2, k) \leq .5 \log (108) k / \log k(\approx 2.341 k / \log k)$ are bounded and so, using the Prime Number Theorem again, it follows that the $k$ satisfying (14) are bounded.

7. Proof of Theorem 3. The key ingredient in the proof of Theorem 3 is Lemma 10. The proof exploits the idea of the proof of Lemma 7. This time, however, we are a bit more careful in estimating. We use some explicit estimates for $\pi(x)$ and $\theta(x)$ of simple form due to Rosser and Schoenfeld. Here $\theta(x)$, Chebyshev's $\theta$-function, is defined by $\theta(x)=\sum_{p \leq x} \log p$.

LEMMA 9.

(i) $\pi(x)<1.2551 \frac{x}{\log x}$.

(ii) $\frac{x}{\log x}\left(1+\frac{1}{2 \log x}\right)<\pi(x)<\frac{x}{\log x}\left(1+\frac{3}{2 \log x}\right)$ for $x \geq 59$.

(iii) $|\theta(x)-x|<\frac{x}{40 \log x} \quad(x \geq 678407)$.

Pr o of. Proofs of part (i) and (ii) can be found in [RS1] and that of (iii) in $[\mathrm{RS} 2]$.

Let $P(n)$ denote the greatest prime factor of $n$.

Lemma 10. Let $d \in N$ and $\varepsilon>0$ be arbitrary and fixed. If there exists an effectively computable constant $m$ such that

(15a) $\quad(\log \alpha(d)-1-\varepsilon) m$

$$
\geq 2.512 \sqrt{(m+1) d}+2 \log d+\log (m+1)+\log (m d) \omega(d)
$$

and for every $k \geq m$,

$$
\theta(k) \leq(1+\varepsilon) k,
$$


then the solutions $(a, d, k)$ of $\omega(a, d, k) \leq \pi(k)$ with $a<d$ and $k \geq 4$ satisfy $k \leq \max \{P(d), m-1\}$ and can be effectively determined.

Proof. Denote the third product in the right hand side of (13) by $Q$. Suppose $k \geq m$. Using Lemma 9(i) and $\theta(k) \leq(1+\varepsilon) k$ we find

$$
\begin{aligned}
\log P_{k}(a, d) & \leq(\pi(\sqrt{(k+1) d})+1) \log [(k+1) d]+\theta(k)+\log Q \\
& \leq 2.512 \sqrt{(k+1) d}+(1+\varepsilon) k+\log (k+1)+\log d+\log Q .
\end{aligned}
$$

From the latter estimate and Lemma 6(iii) it follows that

$$
\log Q>(\log \alpha(d)-1-\varepsilon) k-2.512 \sqrt{(k+1) d}-2 \log d-\log (k+1) .
$$

The derivative of the right hand side of (15a) with respect to $k$ equals $1.256 d / \sqrt{(k+1) d}+1 /(k+1)+\omega(d) / k$ and on using (15a) and $k \geq m$, we find that the derivative is

$$
\leq \frac{1.256 \sqrt{m d \frac{m}{m+1}}+\frac{m}{m+1}+\omega(d)}{m}<\log \alpha(d)-1-\varepsilon
$$

and is less than the derivative of the left hand side. Thus

$$
(\log \alpha(d)-1-\varepsilon) k \geq 2.512 \sqrt{(k+1) d}+2 \log d+\log (k+1)+\log (k d) \omega(d)
$$

and so

$$
Q=\prod_{\substack{p>k \\ p \mid D_{a, d, k}}} p>(k d)^{\omega(d)}
$$

Now assume that $k>P(d)$ and that $(a, d, k)$ is a solution of $\omega(a, d, k) \leq$ $\pi(k)$. Then the contribution of the primes exceeding $k$ to $\omega(a, d, k)$ exceeds $\omega(d)$ by (16). Taking also into account the contribution of the primes $\leq k$, which is $\pi(k)-\omega(d)$, we conclude that $\omega(a, d, k)>\pi(k)$. This contradiction shows that $k \leq \max \{P(d), m-1\}$. Using this estimate and (1), it follows that the solutions $(a, d, k)$ with $a<d$ can be effectively determined.

Remark 1. It is rather straightforward how to work with this lemma in practice. For a given $d$ we try to calculate a number $k(d)$ satisfying $(15)$, where the numbers in (15a) are rounded off in such a way that if $k$ satisfies the inequalities with the rounded off quantities, this still implies that $k$ satisfies (15a). For $\alpha=1, \ldots, d$ with $(\alpha, d)=1$, we write down $\alpha, \alpha+d, \alpha+$ $2 d, \ldots, \alpha+k(d) d$ and factorize these numbers. Whenever we stumble on a prime factor not previously encountered, we put it "on the stack" (for each $\alpha$ we start with an empty stack). If $i$ terms of the progression are treated in this way, compare the cardinality of the stack $n$, say, with $\pi(i)$. If (and only if) $n \leq \pi(i)$ store $(\alpha, d, i)$; it is a solution of $\omega(a, d, k) \leq \pi(k)$. If $i>k(d)$ stop; we have found all solutions with $a \equiv \alpha(\bmod d)$. 
Remark 2. Erdös [E] used the function $P_{k}(a, d)$ to obtain results on Bertrand's Postulate for arithmetic progressions. In [Mo1] his method is worked out further in this context.

Proof of Theorem 3 . Assume $(a, d, k)$ is a solution of

$$
\omega(a, d, k) \leq \pi(k) .
$$

Then $\omega(a, d, k) \leq \pi(k) \leq k-2$.

In first instance we can restrict ourselves to the case where $k \in K:=\{k$ : $k>3, k=4$ or $k$ is prime $\}$. Whenever $k \notin K$, let $l$ be the largest number $<k$ which is in $K$. Then

$$
\omega(a, d, l) \leq \omega(a, d, k) \leq \pi(k)=\pi(l) .
$$

So to any solution with $k \notin K$ corresponds a solution with $k$ in $K$. Furthermore, if $(a, d, k)$ is a solution with $k \in K$, then it extends at most to finitely many easily computable other solutions $(a, d, k+i)$, with $1 \leq i<k_{1}-k$, where $k_{1}$ is the smallest prime exceeding $k$.

If $k=4$ one finds $a(a+d) \leq 6$ by (1). This leaves four potential solutions $(a, d, 4)$, none of which satisfies (17). Next assume that $k$ is a prime $\geq 5$. Using (7) it follows that

$(k-1-\pi(k)) ! d^{k-1-\pi(k)}<a(a+d) \ldots(a+(k-1-\omega(a, d, k)) d) \leq(k-1) !$ and so

$$
d^{k-1-\pi(k)}<(k-1) \ldots(k-\pi(k)) .
$$

So $d$ is bounded for fixed $k$. In Table 1 below for each $5 \leq k \leq 19, k$ prime, the maximal value of $d$ satisfying (18) is recorded. First assume $k \geq 23$. Using Lemma 9(i) it follows that

$$
\frac{1+\pi(k)}{k}<\frac{1.256}{\log k}+\frac{1}{k}<.45
$$

and so by (18),

$$
\log d<\frac{\pi(k) \log k}{k-1-\pi(k)}<\frac{1.256}{\left(1-\frac{1+\pi(k)}{k}\right)}<2.29
$$

and therefore $d<9.9$. For $k=11,13,17$ and 19 the inequality $d \leq 9$ follows from Table 1. So either $k=5$ or $k=7$ or $2 \leq d \leq 9$. For $k=5$ or $k=7$ we have respectively $a(a+d) \leq 24$ and $a(a+d)(a+2 d) \leq 120$ by the Erdös inequality (7). There are 29 respectively 33 pairs $(a, d)$ with $a$ and $d$ coprime and $d>1$, satisfying these inequalities. For each of them we then check whether $(17)$ is satisfied. We find that $(a, d, k)=(1,2,5)$ is the only solution of (17) with $k=5$ or 7 . Hence we may assume from now on that $k \geq 11$. 
Table 1

\begin{tabular}{|c|c|c|}
\hline$k$ & $\pi(k)$ & Maximum value of $d$ \\
\hline 5 & 3 & 23 \\
\hline 7 & 4 & 18 \\
\hline 11 & 5 & 7 \\
\hline 13 & 6 & 9 \\
\hline 17 & 7 & 7 \\
\hline 19 & 8 & 8 \\
\hline
\end{tabular}

Table 2

\begin{tabular}{|c|c|c|c|c|c|}
\hline$d$ & $\omega(d)$ & $\alpha(d)$ & $\log \alpha(d)$ & $k(d)$ & $d \cdot k(d)$ \\
\hline 2 & 1 & 4.000 & 1.386 & 142 & 284 \\
\hline 3 & 1 & 5.196 & 1.647 & 80 & 240 \\
\hline 4 & 1 & 8.000 & 2.079 & 42 & 168 \\
\hline 5 & 1 & 7.476 & 2.011 & 55 & 275 \\
\hline 6 & 2 & 20.78 & 3.033 & 24 & 144 \\
\hline 7 & 1 & 9.681 & 2.270 & 48 & 336 \\
\hline 8 & 1 & 16.00 & 2.772 & 30 & 240 \\
\hline 9 & 1 & 15.58 & 2.745 & 34 & 306 \\
\hline
\end{tabular}

Table 3

\begin{tabular}{|c|c|c|c|c|}
\hline$k$ & $\alpha+d(k-1)$ & $\omega(a, d, k)$ & $\pi(k)$ & Successive additions to stack \\
\hline 11 & 61 & 9 & 5 & $7,13,19,5,31,37,43,11,61$ \\
\hline 13 & 73 & 11 & 6 & 67,73 \\
\hline 17 & 97 & 14 & 7 & $79,17,97$ \\
\hline 19 & 109 & 16 & 8 & 103,109 \\
\hline 23 & 133 & 18 & 9 & 23,127 \\
\hline
\end{tabular}

For $d=2, \ldots, 9$ the computable constant $m$ of Lemma 10 is bounded above by $k(d)$. These numbers are recorded in Table 2 . In computing them the result that (15b) holds with $\varepsilon=.0011$ for $k \geq 1$ ([RS2]) was used. Carrying through the procedure given in Remark 1 following Lemma 10, one finds that there are no solutions with $a<d, 11 \leq k \leq k(d)-1$, and $2 \leq d \leq 9$. Using Lemma 10 we conclude that there are no solutions with $a<d, k \geq 11$ and $2 \leq d \leq 9$ and so, reasoning as in the proof of Lemma 8 , no solutions with $a \leq k+1, k \geq 11$ and $2 \leq d \leq 9$. Some details of the calculations are given in Table 3 . It remains to find the solutions with $a \geq k+2, k \geq 11$ and $2 \leq d \leq 9$.

First we assume that $a \geq k+2, k \geq 11$ and $d=2$. Using the sharp form of the Erdös inequality (6) we find that

$$
(3 k-2 \pi(k)) ! \leq(k !)^{3} k^{-2} \theta_{d}(k)^{-2} .
$$


Using Lemma 9 (i) it follows that $3 k-2 \pi(k) \geq 2 k$, since $k \geq 11$. On using that

$$
\theta_{2}(k) \geq 2^{[(k-1) / 2]+[(k-1) / 4]+[(k-1) / 8]} \geq 2^{3 k / 4-2}
$$

and the Stirling inequalities in the form

$$
k^{k} e^{-k}<k !<k^{k+1} e^{-k}
$$

it follows that

$$
\frac{9}{2} \log 2-\frac{2 \pi(k) \log k}{k} \leq(\log 2-1) \frac{2 \pi(k)}{k}+\frac{\log (16 k)}{k} .
$$

(Use that $(3 k-2 \pi(k)) ! \geq(3 k-2 \pi(k)) \log (3 k-2 \pi(k))-3 k+2 \pi(k) \geq$ $(3 k-2 \pi(k)) \log (2 k)-3 k+2 \pi(k)$.) This is false by Lemma $9(\mathrm{i})$, since $k \geq 11$.

Finally assume that $a \geq k+2, k \geq 11$ and $d \geq 3$. Using the Erdös inequality we find that

$$
a(a+3) \ldots(a+(k-1-\pi(k)) 3) \leq(k-1) !
$$

for $a=k, k+1$ and $k+2$. On multiplying these three inequalities together and multiplying both sides by $(k-1)$ !, we find that

$$
(4 k-1-3 \pi(k)) ! \leq\{(k-1) !\}^{4}=(k !)^{4} / k^{4} .
$$

Using Lemma 9 (i) it follows that $4 k-1-3 \pi(k) \geq 2.65 k$ for $k \geq 19$. Then proceeding as above it follows that

$$
4 \alpha-\frac{3 \pi(k) \log k}{k} \leq \frac{3 \pi(k)}{k}(\alpha-1)+\frac{\log k-1+\alpha}{k} \quad(k \geq 19)
$$

where $\alpha:=\log (2.65)$. On using Lemma 9 (i) it follows that $k \leq 17$. For the three remaining primes 11, 13 and 17 we check (19) directly. It is found that none of them is a solution.

On gathering the results of all cases, we arrive at the conclusion that $(1,2,5)$ is the only solution of $(17)$ with $k=4$ or a prime $\geq 5$. It is easily seen that the solution $(1,2,5)$ does not extend to further solutions (cf. the beginning of the proof). (Since $\omega(1,2,5)=4=\pi(5)$, the only candidate is $(1,2,6)$ which does not satisfy (17).) So Theorem 3 is proved.

Concluding remark. In this paper we limited ourselves to solving (17). In [Mo2, Chapter 7] the more general problem of solving $\omega(a, d, k) \leq$ $f(k)$ is considered, where $f: \mathbb{N} \rightarrow \mathbb{N}$ is a non-decreasing function. In that case Lemma 10 holds true with $\pi(k)$ replaced by $f(k)$ and the condition $k \geq 4$ by $k \in K_{f}:=\{k: k \geq 2$ and $f(k) \leq k-2\}$. In solving (17) we first solved for the $k$ in $\{k>3: k=4$ or $k$ is prime $\}$, in general this set has to be replaced by $\left\{k \in K_{f}: f(k) \neq f(k-1)\right.$ or $\left.k-1 \notin K_{f}\right\}$.

Acknowledgements. This is a completely rewritten, slightly shortened, version of [Mo2, Chapter 7]. The author is indebted to the referee and especially to Prof. R. Tijdeman for their large contribution in rewriting. The 
latter and Dr. J.-H. Evertse he wishes to thank for proofreading the (many) earlier versions respectively the thesis version and for the helpful remarks and suggestions they made as a result thereof.

\section{References}

[E] P. Erdős, Über die Primzahlen gewisser arithmetischer Reihen, Math. Z. 39 (1934), 473-491.

[G] A. Granville, Integers, without large prime factors, in arithmetic progressions, I, Acta Math. 170 (1993), 255-273.

[Mc1] K. S. McCurley, Explicit estimates for $\theta(x ; 3,1)$ and $\psi(x ; 3,1)$, Math. Comp. 42 (1984), 287-296.

[Mc2] - Explicit estimates for the error term in the prime number theorem for arithmetic progressions, ibid., 265-285.

[Mo1] P. Moree, Bertrand's Postulate for primes in arithmetical progressions, Comput. Math. Appl. 26 (1993), 35-43.

[Mo2] - Psixyology and diophantine equations, Ph.D. thesis, Leiden University, 1993.

[RRu] O. Ramaré and R. Rumely, Primes in arithmetic progressions, Math. Comp., to appear.

[Ri] P. Ribenboim, The Book of Prime Number Records, Springer, New York, 1989 (2nd ed., 1990).

[RS1] J. B. Rosser and L. Schoenfeld, Approximate formulas for some functions of prime numbers, Illinois J. Math. 6 (1962), 64-94.

[RS2] - - - Sharper bounds for Chebyshev functions $\theta(x)$ and $\psi(x)$, Math. Comp. 29 (1975), 243-269.

[Ru] R. Rumely, Numerical computations concerning the ERH, ibid. 62 (1993), 415440.

[ST1] T. N. Shorey and R. Tijdeman, On the number of prime factors of an arithmetical progression, Sichuan Daxue Xuebao 26 (1989), 72-74.

[ST2] - - - On the number of prime factors of a finite arithmetical progression, Acta Arith. 61 (1992), 375-390.

[ST3] - - - On the product of terms of a finite arithmetic progression, in: Proc. Conf. Diophantine Approximations and Transcendence Theory, Y.-N. Nakai (ed.), RIMS Kokyuroku 708, Kyoto, 1990, 51-62.

[ST4] - - - On the greatest prime factor of an arithmetical progression III, in: Proc. Conf. Luminy Transcendence Theory, 1990, Ph. Philippon (ed.), de Gruyter, Berlin, 1992.

DEPARTMENT OF MATHEMATICS

PRINCETON UNIVERSITY

FINE HALL - WASHINGTON ROAD

PRINCETON, NEW JERSEY 08544-1000

U.S.A.

E-mail: MOREE@RULFC1.LEIDENUNIV.NL 